\title{
BMJ Open A phase III randomised clinical trial of perioperative therapy (neoadjuvant chemotherapy versus chemoradiotherapy) in locally advanced gallbladder cancers (POLCAGB): study protocol
}

Reena Engineer, ${ }^{1}$ Shraddha Patkar, ${ }^{2}$ Shirley Christabel Lewis, ${ }^{1}$
Ashutosh Das Sharma, ${ }^{1,3}$ Nitin Shetty, ${ }^{3}$ Vikas Ostwal, ${ }^{4}$ Anant Ramaswamy, ${ }^{4}$
Supriya Chopra, ${ }^{1}$ Archi Agrawal, ${ }^{5}$ Prachi Patil,,${ }^{6}$ Shaesta Mehta, ${ }^{6}$ Mahesh Goel ${ }^{2}$

\section{ABSTRACT}

Introduction Neoadjuvant chemotherapy (NACT) is considered the current standard for locally advanced gallbladder cancer (GBC). There is no consensus on the optimal neoadjuvant approach. A pilot study from our institution has shown improved overall survival (OS) and progression-free survival (PFS) with neoadjuvant chemoradiation (NACRT). The present randomised phase III trial is designed to compare NACRT with NACT alone and will test the superiority of chemoradiation in terms of tumour downstaging and improvement in OS.

Methods and analysis Patients with biopsyproven locally advanced GBC (T3-4) with predefined clinical-radiological features will be randomised to the gemcitabine-based chemotherapy-alone arm or the chemoradiation arm. Patients with resectable disease or with distant metastases will be excluded. The primary end point of the study is to compare OS between the two arms. The secondary end point was to compare PFS, R0 resection rates, acute and late toxicity, postoperative complications and quality of life between the two study arms. The trial is designed to detect an improvement in median 0 S by 5.5 months in the study arm (11 months in the control group, HR of 0.7 ) with $80.0 \%$ power at a 0.05 significance level. The resultant sample size to achieve this aim is 314 ( 157 in each arm) over a duration of 5 years with a $10 \%$ attrition rate.

Ethics and dissemination The institutional ethics committee has approved this trial and will be routinely monitoring the trial at frequent intervals. The results of the study will be disseminated via peer-reviewed scientific journals, conference presentations and submission to regulatory authorities.

Registration The trial is registered with Clinical Trials Registry India (CTR//2016/08/007199) and ClinicalTrials. gov (NCT02867865). This trial aims to assess the superiority of NACRT over NACT in locally advanced GBCs in terms of improvement in OS. The results of this study will define the optimal neoadjuvant approach in locally advanced GBC.
Strengths and limitations of this study

- This is the first randomised study evaluating chemoradiation in the neoadjuvant setting.

- Treatment of locally advanced gallbladder cancer $(\mathrm{GBC})$ is not standardised, and this trial will give an opportunity to do so.

- Slower recruitment of patients: as majority of patients with GBC in the population present late in the course of the disease, a large fraction of the screened patients turn out to be metastatic or with advanced disease that do not meet the stringent inclusion criteria for the trial. This has resulted in low enrolment into the study.

- Compliance of patients: the long treatment time (>6 months), combined with the socioeconomic restrictions of the majority of the population, makes it challenging for the patients to stick to the advised care, resulting in financial burden and subsequently increased susceptibility of dropout and loss of follow-up.

- As the treatment is decided and delivered by a large team of physicians that consists of radiologists, gastroenterologist, oncosurgeons, medical oncologists and radiation oncologists, the coordination of the team becomes challenging.

Trial registration number NCT02867865; Pre-results.

\section{INTRODUCTION}

Gallbladder cancer (GBC) is the most common malignancy of the biliary tract. ${ }^{1}$ Its incidence is alarmingly high in Chile, Japan and northern India. ${ }^{2}$ Complete surgical excision is the standard of care for early-stage GBC. Unfortunately, majority of the cases are diagnosed at an advanced or metastatic stage, 
and only $10 \%-30 \%$ of the patients present with resectable disease. $^{2}$

GBC with liver infiltration, with or without visceral/ vascular infiltration, or having large local lymph node metastases in the absence of distant metastases is generally considered as locally advanced. Prognosis for locally advanced disease in terms of resectability and survival remains dismal in most of the reports. ${ }^{3}$ Even with aggressive surgery like extrahepatic bile duct resection or pancreaticoduodenectomy, the 5-year survival rate for stage III disease at best ranges from $30 \%$ to $42 \%$. These results are often not reproducible in routine clinical practice. $^{4-9}$

Locally advanced GBC, where surgery is not possible, is treated with chemotherapy alone as the current standard of care. In the advanced biliary cancer (ABC-02) trial by Valle et al, neoadjuvant chemotherapy (NACT) with gemcitabine and cisplatin was found to be superior to gemcitabine alone in terms of local tumour response. ${ }^{10}$ Some locally advanced non-metastatic GBCs do get downstaged to undergo resection following NACT. In a publication from our institute, gemcitabine/cisplatin-based NACT alone resulted in an R0 resection rate of $46 \%$ and a median overall survival (OS) and progression-free survival (PFS) of 13.4 and 8.1 months, respectively. ${ }^{11}$

Few studies have reported the outcomes of neoadjuvant chemoradiation (NACRT) with limited success. ${ }^{12} 13$ In an earlier report, we published the outcomes of three patients with unresectable tumours, two of which were downstaged to undergo R0 resection with high-dose NACRT. ${ }^{14}$ In a pilot study of 28 patients treated with NACRT conducted at our centre, $47 \%$ of the patients underwent R0 resections with median OS and PFS being 35 and 20 months, respectively. ${ }^{15}$

There is no consensus on the optimal neoadjuvant approach in locally advanced gallbladder carcinoma (GBC). However, most of the treating physicians prefer to use NACT alone followed by surgical resection if downstaging is achieved. The present randomised trial is designed to compare NACRT with NACT alone and will test the superiority of one over the other in terms of downstaging and prolonging survival.

\section{METHODS AND ANALYSIS Hypothesis}

On the basis of encouraging results obtained with NACRT, we hypothesise that NACRT and additional chemotherapy will improve OS compared with NACT alone in locally advanced GBC.

\section{Study aim}

The primary aim of the study is to compare the OS between the patients treated with NACT alone with NACRT. The secondary aim is to compare the R0 surgical resection rate, PFS, acute and late toxicities, postoperative complications and quality of life (QOL) between two study arms.

\section{Study design}

This study is a phase III randomised control trial designed to compare the OS between the two neoadjuvant treatment arms. All patients with diagnosis of non-metastatic locally advanced GBC who fulfil the study eligibility criteria will be evaluated for participation in the study. Patients will undergo upfront randomisation into one of the study arms (NACT or chemoradiation) using permuted block stratified randomisation. Stratification will be done according the T stage (T1-4). All randomisations will be done through the clinical research secretariat at Tata Memorial Hospital (TMH).

\section{Research setting}

The study will be conducted at TMH, Tata Memorial Centre, and Advanced Centre for Treatment Research and Education, Mumbai, India, and other collaborating centres.

\section{Patients and public involvement}

This research was conceived without patient and public involvement. The patients and the public were not invited at any stage of the study design or initiation.

\section{Screening}

All patients will be screened for distant metastases at baseline (prior to randomisation) using positron emission tomography-contrast-enhanced CT (PET-CECT) scan. Patients found to be non-metastatic will be subjected to staging laparoscopy to rule out peritoneal metastases. A tissue diagnosis from the primary would be done by either biopsy or fine needle aspiration cytology.

\section{Participants eligibility}

\section{Inclusion criteria}

Patient older than 18 years of age with histologically proven diagnosis of locally advanced GBC (adenocarcinoma) $\mathrm{T} 3$ or $\mathrm{T} 4$ tumours with one or more of the following criteria will be included in the trial:

1. Liver invasion: more than $2 \mathrm{~cm}$ but less than $5 \mathrm{~cm}$.

2. Radiological involvement of the antropyloric region of the stomach, duodenum, hepatic flexure of the colon or small intestine, but without infiltration of the mucosa on endoscopy.

3. Type I/II invasion: involvement of the bile duct (common hepatic duct or proximal one-third of the common bile duct) on percutaneous transhepatic biliary drainage/magnetic resonance cholangiopancreatography causing obstructive jaundice.

4. Radiological suspicion of regional lymph node involvement along the hepatic artery, hepatoduodenal ligament, retropancreatic/retroduodenal: size $>1 \mathrm{~cm}$ in short axis, round in shape and heterogeneous enhancement on PET scan.

5. Vascular involvement: impingement/involvement ( $<180$ degree angle) of one or more of the following blood vessels: common hepatic artery/right hepatic artery/main portal vein/right portal vein (stage III disease). 
6. Patients who have undergone prior cholecystectomy, having residual disease with at least one of the above features.

7. The patients must have good general condition Eastern Co-operative Oncology Group (ECOG 0-2).

8. Normal haematological, renal and hepatic functions done no earlier than 2 weeks prior to treatment initiation.

\section{Exclusion criteria}

Those patients with resectable disease or with evidence of distant metastasis (liver, lung, peritoneum and port site) are excluded from the study. Patients with involvement of the major part of the liver that restricts delivery of full radiotherapy (RT) doses and those who have undergone prior radiation or chemotherapy are deemed ineligible to participate in the trial. Patients with evidence of active cholangitis or unresolved biliary obstruction will be excluded.

\section{Informed consent}

Any of the investigators or coinvestigators or a research officer/nurse of this trial can obtain informed consent or assent from potential trial participants or authorised surrogates. The consent form will be given at least 2 days prior to randomisation. All efforts would be taken to keep their confidentiality.

\section{Study interventions}

NACT-alone arm (standard arm)

Patients randomised to NACT-alone arm will proceed to receive four cycles of gemcitabine $\left(1000 \mathrm{mg} / \mathrm{m}^{2}\right)$ delivered on days 1 and 8 every 3 weeks, along with cisplatin $\left(25 \mathrm{mg} / \mathrm{m}^{2}\right)$ on day 1 . Patients will be assessed for response and evaluated for surgical resection using PET-CECT scan after four cycles of chemotherapy. If the scans show stable but unresectable disease, then patients will continue to undergo the same chemotherapy. Patients showing locally progressive/systemic disease may be considered for either second-line palliative chemotherapy or best supportive care. The use of radical-dose chemoradiation is not allowed on disease progression in this arm. However, palliative radiation of 20-25 Gy in four to five fractions may be used.

Prior to each cycle of chemotherapy, assessment of haematological, renal and hepatic functions will be done. Patients with creatinine clearance $>50 \mathrm{~mL} / \mathrm{min}$ cisplatin will be administered at a dose of $25 \mathrm{mg} / \mathrm{m}^{2}$. In case of creatinine clearance between 40 and $50 \mathrm{~mL} / \mathrm{min}$, either substitution with oxaliplatin or $20 \%-25 \%$ dose reduction may be applied. Gemcitabine+cisplatin or gemcitabine+oxaliplatin is used and dose modification is done as decided by the medical oncologist's decision as per standard oncological guidelines.

\section{NACRT arm (experimental arm)}

Patients randomised to the NACRT arm will undergo radiation therapy for 5 weeks with concurrent gemcitabine-based chemotherapy $\left(300 \mathrm{mg} / \mathrm{m}^{2}\right.$ weekly) followed by two cycles of gemcitabine+cisplatin systemic chemotherapy. The dose and schedule for gemcitabine+cisplatin chemotherapy will be same as the standard arm. The chemotherapy will start 3 weeks after completion of radiation.

\section{RADIOTHERAPY PLANNING AND CONTOURING Simulation}

The planning contrast-enhanced CT (CECT) scan will be done in fasting state with the patient in supine position with arms over the head and a knee rest. An immobilisation device such as a vacuum bag or a thermoplastic mask will be used. Fiducials will be placed at the laser intersection points at the level of the xiphisternum. The scan will be taken from the level of the carina to L4-5 levels with $2.5 \mathrm{~mm}$ interslice thickness in the portal phase of intravenous contrast. Respiratory motion management technique, such as Four-dimensional computed tomography (4DCT) or deep inspiratory breath hold technique, may be considered.

\section{Contouring}

The gross tumour volume will be delineated using the information from all available imaging, such as the diagnostic triphasic CECT and PET scan, and it will be fused with planning scan. It will include the primary and involved locoregional lymph nodes. The clinical target volume (CTV) consists of the adjacent areas of suspected microscopic disease in the surrounding liver parenchyma and the draining locoregional lymph nodes at the pericoledochal, cystic duct, retroportal, along the common hepatic artery, along the hepatoduodenal ligament, pancreaticoduodenal, hilar, periportal, portacaval and retropancreatic regions. The planning target volume (PTV) will be generated by adding a safety margin of 5-7 mm around the CTV to countermotion and set-up variations. The aim will be to deliver $52-57 \mathrm{~Gy} / 25$ fractions to the gross disease and $45 \mathrm{~Gy} / 25$ fractions to the suspected microscopic disease, along with weekly gemcitabine $\left(300 \mathrm{mg} / \mathrm{m}^{2}\right)$.

Organ at risk (OAR) contouring will include the liver, oesophagus, stomach, duodenum, kidneys, heart, lungs, bowel and spinal cord. All care will be taken to restrict OAR doses as per standard guidelines. The dose constraints for the OAR are as follows: $70 \%$ of the liver $<30 \mathrm{~Gy}$, mean liver dose $<25 \mathrm{~Gy}, 70 \%$ of each kidney to receive $<20 \mathrm{~Gy}$, mean dose $<18 \mathrm{~Gy}$ for each kidney, V15 of the small bowel $<190 \mathrm{cc}$ and for the duodenum: V $55<1 \mathrm{cc}$, V50<4cc. ${ }^{16}$ Special focus will be given to restrict dose to normal liver parenchyma and adjacent gastrointestinal structures, such as the duodenum, to minimise radiation-induced grade 3 or higher toxicity.

\section{Radiation plan}

All patients will be treated with intensity-modulated radiotherapy (IMRT) (RapidArc or tomotherapy). Dosevolume histograms will be evaluated for target volume 
coverage and normal tissue-sparing according to standard IMRT plan evaluation indices. ${ }^{17}$ It will be ensured that $95 \%$ of the target volume receives at least $95 \%$ of the prescribed dose. Volumes of hotspots $(>107 \%$ of the prescription dose) will the kept as low as possible throughout the treatment volume. Patient-specific quality assurance of the approved dose plan will be done prior to starting RT.

\section{Treatment delivery and monitoring}

Treatment will be delivered with daily megavoltage/kilovoltage CT imaging to ensure adequate PTV coverage. All patients will be prescribed prophylactic antacids and mucosal coating agent from day 1 of radiation starting as a measure to prevent duodenal toxicity. Haematological, hepatic and renal functions, as well as tolerance to the treatment, will be assessed weekly during NACRT. Toxicity will be recorded as per National Cancer Institute Common Terminology Criteria (CTCAE) V.4.2 at baseline and weekly during NACRT.

\section{Efficacy and safety assessments}

During weeks 12-13 of starting the treatment, PET-CECT scan will be repeated and compared with the initial scans for response assessment using Response Evaluation Criteria in Solid Tumours (RECIST) V.1.1. ${ }^{18}$ The response of the therapy will be assessed in terms of

Complete response (CR): disappearance of all target lesions. Any pathological lymph nodes (whether target or non-target) must have reduction in short axis to $<10 \mathrm{~mm}$.

Partial response (PR): at least a $30 \%$ decrease in the sum of diameters of target lesions.

Progressive disease (PD): at least a $20 \%$ increase in the sum of diameters of target lesions or appearance or one or more lesions. In addition to the relative increase of $20 \%$, the sum must also demonstrate an absolute increase of at least $5 \mathrm{~mm}$.

Stable disease (SD): neither sufficient shrinkage to qualify for PR/CR nor sufficient increase to qualify for PD.

\section{Surgery}

All patients with a CR, PR or SD will be re-evaluated for feasibility of surgery after at least 3 weeks of completion of all neoadjuvant therapy in both arms. Surgical resection, if considered feasible, will be done between weeks 14 and 15 after starting the neoadjuvant therapy. The decision regarding surgery will be taken by a hepatobiliary surgical oncology consultant. Surgical resection will entail en bloc resection of the gallbladder with a wedge excision of liver/segment $\mathrm{IVb} / \mathrm{V}$ excision with an aim to achieve negative margin and complete periportal lymphadenectomy (stations 8,12 and 13), along with a sampling of interaortocaval nodes to detect occult metastasis. Additional organ resection may be performed if necessitated to achieve R0 status as guided by intraoperative frozen section. Performance of extended resections like pancreatoduodenectomy or major hepatectomy to achieve negative margins will be left to the discretion of the operating surgeon. Complications following surgery will be recorded as per the Clavien-Dindo grading system. ${ }^{19}$

\section{Adjuvant therapy}

All the patients after surgery will undergo three cycles of adjuvant chemotherapy with gemcitabine $(1000 \mathrm{mg} /$ $\left.\mathrm{m}^{2}\right)$ on days 1 and 8 and cisplatin $\left(25 \mathrm{mg} / \mathrm{m}^{2}\right)$ on day 1 delivered every 3 weeks. Patients with PD and those with $\mathrm{CR} / \mathrm{PR} / \mathrm{SD}$ and are not eligible for surgery will be evaluated for second-line palliative chemotherapy or best supportive care.

\section{Treatment evaluation}

CTCAE V.4.2 will be used for reporting all acute and late toxicities. Adverse events (AEs) in both arms will be graded using the CTCAE V.4.2. Toxicity profile for thrombocytopenia, neutropenia, fatigue, neuropathy, vomiting, flu-like syndrome, hepatic dysfunction, gemcitabine-induced rash and febrile neutropenia will be recorded. Grade 3 or more thrombocytopenia and vomiting is dose-limiting toxicity and warrants a dose reduction of $25 \%$. If grade 3 or more toxicities are observed during radiotherapy, concurrent gemcitabine dose will be withheld for a week.

\section{Quality of life}

FACT-Hep V.4 will be used to assess QOL scores of all five modules of physical well-being, social/family wellbeing, emotional well-being, functional well-being and hepatobiliary function-related specific questions. It will be done at baseline, at completion of all neoadjuvant treatment (prior to surgery), at treatment completion and at all follow-up time points.

\section{Follow-up}

Patients will be evaluated every 3 months for a period of 2 years with routine complete haemogram and biochemistry, along with ultrasound of the abdomen and pelvis on each follow-up and thereafter every 6 months. QOL with FACT-Hep will be filled on every follow-up.

\section{STATISTICAL CONSIDERATIONS \\ Outcome measures}

The following outcome measures will be recorded.

OS: time interval between the date of the first neoadjuvant treatment and death due to any cause.

PFS: time interval between the date of randomisation and locoregional or distant disease progression or death from any cause.

R0 surgical resection rate: negative surgical margin (R0) rate as determined by intraoperative frozen section. However, final confirmation of the margin status on the histopathology report of the specimen would be done.

R1 resection: microscopic positive margin on histopathology.

R2 resection: presence of gross residual disease or tumour spillage during surgery. 
The number of patients downstaged by neoadjuvant treatment in either arm enabling a margin-negative surgical resection will be documented.

Response rate: response to treatment will be assessed with PET-CECT scan using RECIST as mentioned previously. The pathological response rate in both arms would also be assessed.

\section{Primary endpoint}

The primary end point of the study is the OS. The estimated median OS in the control group is 11 months with an expected increase of median OS of 16.5 months in the study arm. The sample size is 286 subjects (143 in each arm) with $80.0 \%$ power at a 0.05 two-sided significance level to detect an HR of 0.7 when the control group median OS has an HR of 1 . With $10 \%$ expected lost to follow-up in both groups, we will accrue 314 patients (157 in each arm) for the whole study. The study duration is 60 months, of which subject accrual will be done in the first 36 months. The accrual pattern across time periods is uniform (all periods equal). To improve accrual, other centres will be encouraged to participate in this study.

\section{Data collection}

All the data related to the study will be collected and maintained by the principal investigator at the TMH, Mumbai.

\section{Treatment planning data}

The volume, mean, median and maximum radiation dose to PTV, the duodenum, liver, stomach, both the kidneys and the bowel will be recorded for each patient. In addition, V30 liver, V20 kidney, V15 small bowel and V45, V50 and V55 of the duodenum will be recorded.

\section{Treatment data}

Data of all the treatment received will be compiled to report the dose of radiation to target and OAR, overall time of treatment, treatment gaps, if any, chemotherapy dose, dose reductions, surgical outcomes and postoperative complications.

\section{Toxicity evaluation}

Treatment-related toxicity will be reported using CTCAE V.4.2. CTCAE forms will be filled at baseline before starting radiation, weekly during treatment and on each scheduled follow-up. If any toxicity occurs at another time point, additional forms will be filled to record the toxicity.

\section{Quality of life}

FACT-Hep V.4 will be used to assess QOL scores of all five modules of physical well-being, social/family wellbeing, emotional well-being, functional well-being and hepatobiliary function-related specific questions. It will be done at baseline, during treatment (at completion of neoadjuvant treatment), at treatment completion and at subsequent follow-ups.

\section{Clinical outcome data}

The status of the disease will be evaluated with physical examinations and required investigations and will be recorded at each follow-up. A detailed systemic work-up will be performed annually to detect, record and report the locoregional and distant controls.

\section{Protocol compliance}

Inability to receive the planned treatment as per the protocol (chemotherapy as well as radiation) will be regarded as a major violation that will be reported to the institutional review board (IRB). The inability to achieve target or OAR constraints or the patient missing two to three fractions of RT or one to two cycles of concurrent chemotherapy will be considered as a minor violation.

\section{Event reporting}

All events related to the study will be recorded using CTCAE V.4.2. All serious adverse events (SAEs) will be reported. SAEs within the test arm will necessitate hospitalisation. Toxicity arising out of systemic chemotherapy or patients developing cholangitis will not be considered a trial-related injury or a related SAE. CTCAE V.4.2 will be used for reporting of all SAEs to the IRB within 24 hours of the occurrence. Similarly, all deaths will be reported to the IRB.

\section{Trial monitoring}

The progress of the trial will be monitored at regular intervals (annually) by the institutional data and safety monitoring board, and the report will be submitted to the ethics committee and the IRB. The process will be independent of investigators and the sponsor.

\section{Data analysis plan}

No interim analyses have been planned for this study; however, the data monitoring committee has full authority to stop the trial if it perceives harm to any of the arm of patients. Intention-to-treat analysis will be performed along with survival for patients who undergo surgery in both groups.

\section{Primary aim}

Kaplan-Meier curves for OS will be generated for both arms, and OS will be compared using log-rank test stratified for the stratification factors that were used during randomisation (T stage). A $p$ value of $<0.05$ will be considered statistically significant and will be used to reject the null hypothesis.

\section{Secondary aim}

A similar Kaplan-Meier analysis will be performed for PFS. Toxicity assessment will be using categorised groups between the two arms and $\chi^{2}$ test will be used. R0 surgical resection rates and response rates will be calculated within each treatment arm, along with exact 95\% CIs based on binomial distributions compared between treatment arms using two-sample Cochran-Mantel-Haenszel test at the 5\% level of significance. Rates of grades 3 and 4 AEs will be summarised by treatment arm using descriptive statistics. 


\section{Ethics and dissemination}

The institutional ethics committee of TMH, Mumbai, has approved this trial and will be routinely monitoring the trial at frequent intervals. The results of the study will be disseminated via peer-reviewed scientific journals, conference presentations and submission to regulatory authorities.

\section{QOL ANALYSIS}

Standard recommendations will be used to analyse QOL data of the two study arms and repeated measures analysis of variance will be used to compare QOL between the two arms.

\section{Implications for research}

The current study aimed to assess the benefit of NACRT over chemotherapy alone in terms of OS in locally advanced GBC. If proven to be effective, it would redefine the current standard of care for these patients.

\section{Author affiliations}

${ }^{1}$ Department of Radiation Oncology, Tata Memorial Hospital, Mumbai, Maharashtra, India

${ }^{2}$ Department of Surgical Oncology, Tata Memorial Centre, Mumbai, Maharashtra, India

${ }^{3}$ Department of Radiodiagnosis, Tata Memorial Centre, Mumbai, Maharashtra, India ${ }^{4}$ Department of Medical Oncology, Gastrointestinal Disease Management Group, Tata Memorial Centre, Mumbai, Maharashtra, India

${ }^{5}$ Department of Nuclear Medicine, Tata Memorial Hospital, Mumbai, Maharashtra, India

${ }^{6}$ Department of Digestive Diseases and Clinical Nutrition, Tata Memorial Centre, Mumbai, Maharashtra, India

Contributors RE is the principal investigator and participated in the project concept, design, final approval, and manuscript preparation, review and submission. SP participated in the final approval of the protocol and manuscript preparation, review and submission. SCL participated in the review and final approval of the protocol, and manuscript preparation. ADS participated in the manuscript preparation, review and submission. NS participated in the final approval of the draft, and the review and preparation of the manuscript. V0 participated in the review of the manuscript for content, review and preparation of manuscript, submission of the final protocol and final approval. AR participated in the review and preparation of manuscript, and preparation and final approval of the trial protocol. SC participated in the drafting of the final protocol, review of the contents of the protocol, manuscript preparation and final approval. AA participated in the manuscript preparation, review of the final protocol and approval of the final protocol draft. PP participated in the concept and design, preparation and review of the manuscript, and the final approval of the trial protocol. SM participated in the review and preparation of the manuscript, drafting of the final report and final approval. MG (co-principal investigator) participated in the concept and design, review and preparation of the manuscript, drafting of the final report and final approval of the trial protocol.

Funding The study is funded by an intramural grant supported by the institution where it is being conducted.

Competing interests None declared.

Patient consent for publication Not required.

Provenance and peer review Not commissioned; externally peer reviewed.
Open access This is an open access article distributed in accordance with the Creative Commons Attribution Non Commercial (CC BY-NC 4.0) license, which permits others to distribute, remix, adapt, build upon this work non-commercially, and license their derivative works on different terms, provided the original work is properly cited, appropriate credit is given, any changes made indicated, and the use is non-commercial. See: http://creativecommons.org/licenses/by-nc/4.0/.

\section{REFERENCES}

1. de Groen PC, Gores GJ, LaRusso NF, et al. Biliary tract cancers. N Engl J Med 1999;341:1368-78.

2. Misra S, Chaturvedi A, Misra NC, et al. Carcinoma of the gallbladder. Lancet Oncol 2003;4:167-76.

3. Jin LX, Pitt SC, Hall BL, et al. Aggressive surgical management of gallbladder cancer: at what cost? Surgery 2013;154:266-73.

4. Dixon E, Vollmer CM, Sahajpal A, et al. An aggressive surgical approach leads to improved survival in patients with gallbladder cancer: a 12-year study at a North American Center. Ann Surg 2005;241:385.

5. Nishio H, Ebata T, Yokoyama Y, et al. Gallbladder cancer involving the extrahepatic bile duct is worthy of resection. Ann Surg 2011;253:953-60.

6. D'Hondt M, Lapointe R, Benamira Z, et al. Carcinoma of the gallbladder: patterns of presentation, prognostic factors and survival rate. An 11-year single centre experience. Eur J Surg Oncol 2013;39:548-53.

7. Sasaki R, Takahashi M, Funato O, et al. Hepatopancreatoduodenectomy with wide lymph node dissection for locally advanced carcinoma of the gallbladder--long-term results. Hepatogastroenterology 2002;49:912-5.

8. Araida T, Yoshikawa T, Azuma T, et al. Indications for pancreatoduodenectomy in patients undergoing lymphadenectomy for advanced gallbladder carcinoma. $J$ Hepatobiliary Pancreat Surg 2004;11:45-9.

9. Birnbaum DJ, Viganò L, Ferrero A, et al. Locally advanced gallbladder cancer: which patients benefit from resection? Eur J Surg Oncol 2014;40:1008-15.

10. Valle J, Wasan $\mathrm{H}, \mathrm{Palmer} \mathrm{DH}$, et al. Cisplatin plus gemcitabine versus gemcitabine for biliary tract cancer. N Engl J Med 2010;362:1273-81.

11. Chaudhari VA, Ostwal V, Patkar S, et al. Outcome of neoadjuvant chemotherapy in "locally advanced/borderline resectable" gallbladder cancer: the need to define indications. $H P B$ 2018;20:841-7.

12. de Aretxabala X, Losada H, Mora J, et al. Neoadjuvant chemoradiotherapy in gallbladder cancer. Rev Med Chil 2004;132:51-7.

13. Morganti AG, Trodella L, Valentini V, et al. Combined modality treatment in unresectable extrahepatic biliary carcinoma. Int J Radiat Oncol Biol Phys 2000;46:913-9.

14. Engineer R, Wadasadawala T, Mehta S, et al. Chemoradiation for unresectable gall bladder cancer: time to review historic nihilism? $\mathrm{J}$ Gastrointest Cancer 2011;42:222-7.

15. Engineer R, Sastri S, George GJ. Retrospective Study on the Dosimetric and Clinical Evaluation of Duodenal Toxicity in Those Who Underwent Radiation Therapy for Cancers of the Upper Gastrointestinal Tract and Gynecological Cancers Who Received Extended Field Radiotherapy. Int J Radiat Oncol Biol Phys 2017;99:E147.

16. Engineer R, Goel M, Chopra S, et al. Neoadjuvant chemoradiation followed by surgery for locally advanced gallbladder cancers: a new paradigm. Ann Surg Oncol 2016;23:3009-15.

17. Grégoire V, Mackie TR. State of the art on dose prescription, reporting and recording in Intensity-Modulated Radiation Therapy (ICRU report No. 83). Cancer/Radiothérapie 2011;15:555-9.

18. Eisenhauer EA, Therasse P, Bogaerts $\mathrm{J}$, et al. New response evaluation criteria in solid tumours: revised RECIST guideline (version 1.1). Eur J Cancer 2009;45:228-47.

19. Dindo D, Demartines N, Clavien PA. Classification of surgical complications: a new proposal with evaluation in a cohort of 6336 patients and results of a survey. Ann Surg 2004;240:205. 